\title{
利用指数律了解溶液中复杂高分子单链及聚集态结构的形状特征
}

\author{
李涛陆丹* \\ (吉林大学化学学院 超分子结构与材料国家重点实验室＼cjkstart长春 130012)
}

\begin{abstract}
摘要 高分子在溶液中的单链构象和聚集态结构是高分子科学的重要内容, 在研究中往往应用到许多指数经验规律. 总结了几种通常用来研究高分子溶液的指数律方法, 并介绍了指数律如何应用于复杂高分子单链及聚集态结构的形状 特点研究. 通过前体溶液的调控实现对薄膜凝聚态结构以及形貌的操纵是一种新思路、新方法. 因此, 深刻认识高分子 在前体溶液中的本征性质尤为重要. 通过指数律研究, 结合电镜、光谱等方法, 可深入研究高分子溶液单链、聚集尺寸 及形态结构演变与各层次结构单元的运动过程及规律, 为高分子材料的分子设计、功能开发及应用奠定理论基础.
\end{abstract}

关键词 高分子溶液; 指数律; 单链构象; 凝聚态结构; 结构演变

\section{Shape Characteristics of Complex Single Chain and Aggregation by Exponential Law}

\author{
Li, Tao Lu, Dan* \\ (State Key Laboratory of Supramolecular Structure and Materials, College of Chemistry, Jilin University, \\ Changchun 130012)
}

\begin{abstract}
Being an important part of polymer science, the single chain conformation and aggregation structure in polymer solution has been widely studied by many experiential exponential laws. In the review, several kinds of commonly used exponential laws were summarized, and the use in the study of shape characteristics of complex single chain and aggregation was introduced. The aggregation structure and morphology of films can be controlled by precursor solution, so deep understanding to the intrinsic properties of precursor solution is particularly important. Combined with the electron microscope, spectra, etc., the exponential law can be used to further study the single chain, aggregation size and morphology, structure evolution, and the law of the movement process of structure units at all levels in polymer solution, this will lay a theoretical foundation for the molecular designing, functional development and application of polymer materials.
\end{abstract}

Keywords polymer solution; exponential law; single chain; aggregation structure; structural evolution

\section{1 引言}

自 Staudinger 提出高分子的概念以来，高分子科学 和高分子材料工业的发展可谓突飞猛进. 高分子材料成 为材料领域的研究热点, 已在国民经济, 国防科技和尖 端技术等各个领域得到了广泛应用 ${ }^{[1]}$. 高分子溶液是人 们在生产和实践过程中最常遇见的对象, 溶液中高分子 的单链或者凝聚态结构对最终高分子材料成品的结构 与性能往往会有非常大的影响. 因此, 对高分子在溶液 中的基础研究是高分子科学的重要内容.

高分子单链分子一般都有较多的结构单元, 而且分 子量巨大, 结构复杂; 更重要的是, 高分子链还具有自 相似性和分形性的特点. 因此, 它具有其他凝聚态物质 所不具有的标度性. 法国科学家 De Gennes 在其论著 《高分子物理学中的标度概念》中对相关内容给予了深 入阐述 ${ }^{[2]}$. 表征高分子特性的函数可以写成一个系数因 子乘以一个标度形式, 即用一个简单指数方程 $\left(y=k x^{b}\right)$,
将两种不同物理变量建立非线性联系. 其中: 前置系数 $k$ 通常由单体的性质决定, 而由长链决定的物理性质则 出现在标度律中. 幂指数 $b$ 就是标度参数, 通常不等于 1 (等于 1 , 即线性关系) ${ }^{[1]}$. 这一概念的提出得到了科学 界的高度关注, 目前, 已成为高分子科学领域重要且基 础的概念和理论工具. 由于高分子的分子量具有多分散 性，故高分子具有复杂的非线性特征，这使得人们对高 分子的研究步履艰难. 标度律以指数律的形式, 为人们 探究结构和行为均比较复杂的高分子世界的规律提供 了形式简单的理论工具 ${ }^{[3]}$. 更进一步，由于自相似、分形 等概念的引入, 人们发现在高分子溶液聚集方面也存在 较多指数律关系. 因此, 指数律虽是一种简单的数学方 程，但它可直观、深刻的描述高分子的非线性行为，揭 示软物质从无序到有序变化的一般规律. 采用指数律, 可清晰地描述真实分子链的构象及在不同溶液中的形 态; 也可描述在亚浓溶液乃至浓厚体系中分子链聚集状 态在外场刺激下的变化过程及规律. 这对了解高分子链

\footnotetext{
*E-mail: lud@jlu.edu.cn; Tel.: 0431-85167057; Fax: 0431-85193421

Received May 20, 2016; published July 6, 2016.

Project supported by the National Natural Science Foundation of China (Nos. 21174049, 91333103, 21574053).

项目受国家自然科学基金(Nos. 21174049, 91333103, 21574053)资助.
} 
在溶液中的本征性质及其在固态薄膜与高效率功能器 件的应用意义重大 ${ }^{[3]}$.

目前, 越来越多独具特色的高分子材料被发现或合 成, 但人们对其在溶液中的链构象和聚集态结构等本征 性质了解甚微. 事实上, 高分子各种物理性质都与其溶 液性质相关. 因此, 利用指数规律研究高分子在溶液中 的单链及凝聚态形态结构, 揭示其结构的转变、结构单 元的运动规律, 将会加深对高分子物理本质的认识, 构 筑性能更加优良的高分子材料.

本文总结了一些通常用于高分子指数律研究的手 段: 如光散射法、小角中子散射法和黏度法以及指数律 在天然生物、枝化、共轭等高分子溶液中的应用. 包括 这些聚合物在溶液中单链和聚集体的研究.

\section{2 几种常见的利用指数律研究高分子溶液的方 法}

\section{1 黏度法}

一般来说, 当高分子溶液较稀、低于临界接触浓度 时, 溶液中分子链由于相互远离而成为孤立的单链, 此 时溶液黏度性质仅仅与分子量大小有关. 实验证明，当 聚合物、溶剂及温度确定时, 溶液的特性黏度 $[\eta]$ 值仅与 聚合物黏均分子量 $M_{\eta}$ 有关, $[\eta]$ 与 $M_{\eta}$ 的关系符合 MarkHouwink 方程式:

$$
[\eta]=K M_{\eta}^{\alpha}
$$

其中 $\alpha$ 为 Mark-Houwink 指数, 其数值与分子链的形状 有关 ${ }^{[4,5]}$, 可通过测定聚合物溶液的黏度 $[\eta]$ 和黏均分子 量 $M_{\eta}$ 得到, 如图 1 所示. 一些常见的聚合物一溶液体系 的 $\alpha$ 值可在有关手册中查询, 表 1 给出了部分体系的 $\alpha$ 值. 总体而言, 当高分子链是无规柔性线团时, $\alpha$ 值在 $0.5 \sim 0.6$ 之间; 而当高分子链是棒状结构时, $\alpha$ 值大概是 $1.0^{[4]}$.

表 1 Mark-Houwink 指数 $\alpha$ 值对应的分子链形状

Table 1 The molecular chain shape corresponding to the value of Mark-Houwink index $\alpha$

\begin{tabular}{cccc}
\hline $\begin{array}{c}\text { Mark-Houwink } \\
\text { index } \alpha\end{array}$ & 0.33 & $0.5 \sim 0.6$ & 1.0 \\
\hline 分子链形状 & 球状 & 柔性无规线团 & 棒状 \\
\hline
\end{tabular}

Teraoka 等 ${ }^{[5]}$ 的研究认为, 在 $\theta$ 溶剂中, 柔性链的 $\alpha$ $=0.5$; 在良溶剂中, 柔性链的 $\alpha$ 在 $0.7 \sim 0.8$ 之间; 而刚 性链的 $\alpha$ 值则大于 1 . 可见, 当指数 $\alpha$ 越大, 链越伸展. 这是由于同样伸直长度的分子链, 指数大的在溶液中的 运动摩擦力将会更大. 因此, 根据这一规律, 用黏度法 测定不同分子量聚合物在稀溶液中的特性黏度, 再根据 Mark-Houwink 方程得出幂指数 $\alpha$, 可判断分子链在溶液 中的形状.

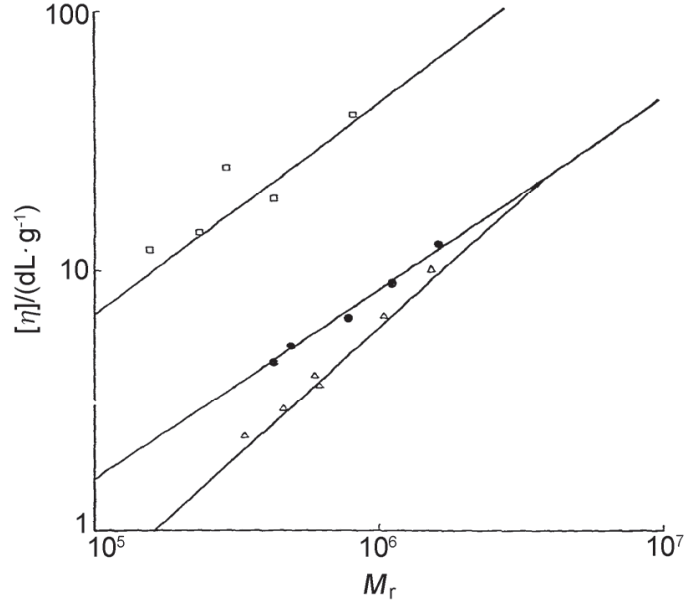

图 1 通过高分子稀溶液中黏均分子量 $M_{\eta}$ 和特性黏度 $[\eta]$ 之间的双对 数图, 得到 Mark-Houwink 指数 $\alpha$

Figure 1 The Mark-Houwink index $\alpha$ was obtained from the double logarithmic plot of intrinsic viscosity $[\eta]$ against viscosity-average molecular weight $M_{\eta}{ }^{[4]}$

\section{2 激光光散射法}

静态光散射法是最常用于高分子溶液表征的方法. 静态光散射法可以测定高分子稀溶液中单链的均方根 回旋半径 $<s^{2}>z^{1 / 2}$, 即 $R_{\mathrm{g}}$. 它是用于高分子溶液研究的重 要参数, 反映了高分子的平均尺寸大小. 对于高分子单 链而言，显然分子量不同时，其在稀溶液中的 $R_{\mathrm{g}}$ 也不 同; 但由于高分子在溶液中的形状并非直链棒状而形态 多样. 因此, $R_{\mathrm{g}}$ 与高分子分子量之间的关系也并非线性, 而是存在如下非线性指数关系:

$$
R_{\mathrm{g}}=k M_{\mathrm{w}}{ }^{v}
$$

其中: 幂指数 $v$ 是分子形状的一个特征参数, 其值反映 了分子链的不同形状, 可通过测定聚合物单链的 $R_{\mathrm{g}}$ 和 重均分子量得到. 如图 2 所示，当指数 $v$ 分别为 0.33 , $0.50 \sim 0.60$ 和 1 时, 分子链的形状是球状、无规线团和 刚性直链 ${ }^{[6]}$. 而它的倒数 $d_{\mathrm{f}}=1 / v$, 也有特殊的物理意 义: $d_{\mathrm{f}}$ 被称为分形维数. 如果 $d_{\mathrm{f}}=1$, 分子链即为棒状直 链; $R_{\mathrm{g}}$ 随着 $M_{\mathrm{w}}$ 线性增加, $d_{\mathrm{f}}=5 / 3 \sim 2$ 时, 高分子链呈现 高斯线团状; 枝化作用会使得 $d_{\mathrm{f}}$ 稍微增加, $d_{\mathrm{f}}=2$ 时, 分 子链呈现二维片状; 而若 $d_{\mathrm{f}}=3$, 则分子链呈现密度分 布均匀的三维球体 ${ }^{[9 \sim 11]}$. 因此, 通过静态光散射测定分 子链的均方根回旋半径 $R_{\mathrm{g}}$, 建立 $R_{\mathrm{g}}$ 与聚合物的重均分 子量 $M_{\mathrm{w}}$ 之间的指数关系得到分形维数 $d_{\mathrm{f}}$, 便可依此判 断分子链在溶液中的形状.

此外，这一公式还可以用于表征溶液中聚集体的形 态结构 ${ }^{[12,13]}$. 聚集体的表观分子量和 $R_{\mathrm{g}}$ 也存在同样的指 数关系, 形状指数 $v$ 的大小代表了聚集的形状. 当 $v=$ $1 / 3$ 时, 聚集体是均匀球状; 当 $v=1 / 2$ 时, 呈无规线团 状; 而当 $v=1$ 时, 聚集体呈直棒状; 其形状与单链一致. 


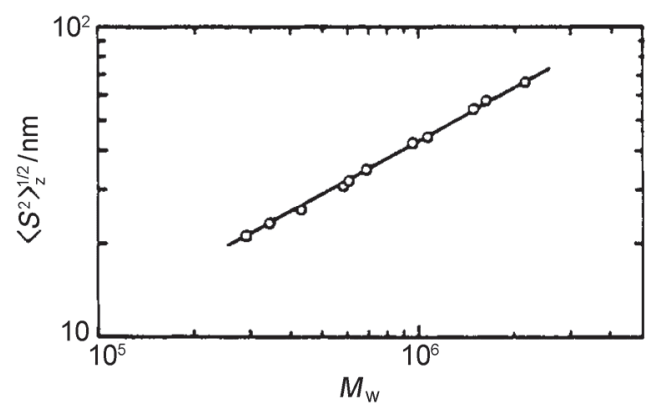

图 2 重均分子量 $M_{\mathrm{w}}$ 和均方根回转半径 $R_{\mathrm{g}}\left(\left\langle s^{2}\right\rangle^{1 / 2}\right)$ 之间的指数关系

Figure 2 Exponential relationship between the root mean square radius of gyration $\left.R_{\mathrm{g}}\left(<\mathrm{s}^{2}\right\rangle^{1 / 2}\right)$ and weight-average molecular weight $M_{\mathrm{w}}{ }^{[6]}$

据报道 ${ }^{[14 ~ 17]}$, 在静态光散射测试过程中, 各角度测 试得到的散射光强度 $I$ 与散射矢量 $q$ 之间也存在着指数 关系:

$$
I \propto q_{\mathrm{f}}^{-d}
$$

其中: $q=(4 \pi n / \lambda) \sin (\theta / 2), d_{\mathrm{f}}$ 就是溶液中粒子的分形维数. 可根据 $d_{\mathrm{f}}$ 的值判断溶液中粒子的形态结构. 如图 3 所示, 其中 $R_{\mathrm{agg}}<q^{-1}<R_{0}\left(R_{\mathrm{agg}} 、 R_{0}\right.$ 分别为聚集和单链的均方根 回旋半径); $d_{\mathrm{f}}$ 则是聚集的分形维数, 可根据 $d_{\mathrm{f}}$ 的值判断 单链和聚集体的形态结构.

由于激光是可见光, 波长一般在几百纳米, 这将导 致散射矢量的倒数 $1 / q$ 比较大(一般远大于高分子单链 的 $R_{\mathrm{g}}$. . 因此, 光散射法多用于表征高分子溶液中的聚 集态结构.

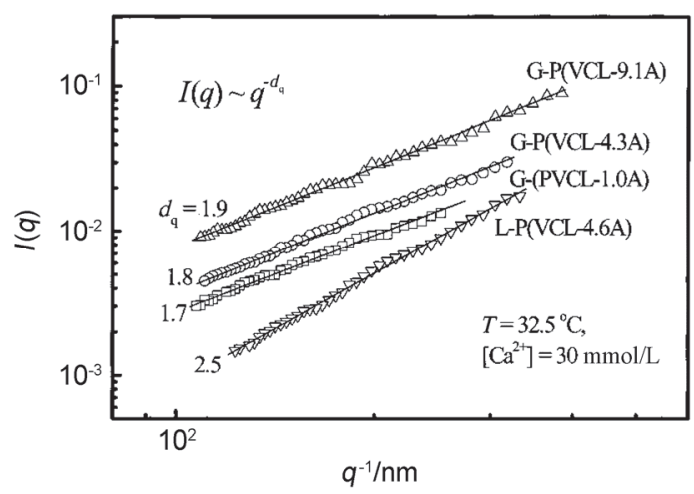

图 3 静态光散射(SLS) 表征光强函数 $I(q)$ 和散射矢量 $q$ 之间的指数关 系

Figure 3 Exponential relationship between the excess scattering intensity $I(q)$ and the scattering vector $q$ by static light scattering (SLS $)^{[17]}$

\section{3 小角中子 $/ X$ 射线散射法}

小角中子 $/ X$ 射线散射法皆是用于高分子溶液研究 的重要手段, 可测定溶液中单链结构和聚集行为 ${ }^{[18 ~ 24]}$, 其测量原理类似于激光光散射法. 如图 4 所示, 各角度 测得的光强与散射矢量同样满足如下指数关系式:

$$
I \propto q_{\mathrm{f}}^{-d_{\mathrm{f}}}
$$

与激光光散射法不同的关键点在于: 中子和 $\mathrm{X}$ 射线 的波长 $(<1 \mathrm{~nm})$ 远远小于激光波长 $(>100 \mathrm{~nm})$, 散射矢
量倒数 $1 / q$ 要远远小于激光光散射的 $1 / q$ 值，故更容易测 量到单链结构. 这两种方法常用于测定各角度的散射光 强 $I$ 与散射矢量 $q$ 之间的指数关系, 而得到单链和聚集 的分形维数, 从而能更深入地研究高分子溶液中单链、 聚集体的形态结构及从单链到聚集的动力学演变过 程 ${ }^{[20 \sim 24]}$.

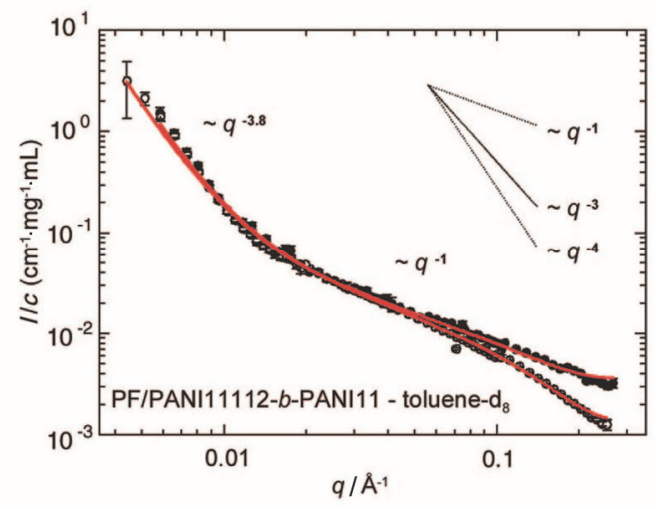

图 4 小角中子散射法中光强函数 $I(q)$ 和散射矢量 $q$ 之间的指数关系 Figure 4 Exponential relationship between the excess scattering intensity $I(q)$ and the scattering vector $q$ by small angle neutron scattering $(\text { SANS })^{[22]}$

\section{4 核磁共振法(NMR)}

结合 Sketjal-Tanner 方程，核磁共振法可用于表征 高分子稀溶液中溶质的平移扩散运动, 从而得到溶液中 粒子的扩散系数 $D$. 在许多研究中人们发现: 如果分子 是均匀分布的, 则其扩散系数 $D$ 与分子量 $M$ 之间将存在 指数关系:

$$
D \propto M^{-\delta}
$$

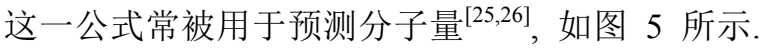
由于此公式在形式上类似于公式(2), 也有很多人认为 $\delta$ 等同于 $v$. Delsuc 等 ${ }^{[27]}$ 专门从实验和理论上对比了这两 个参数的相关性，最终发现当测量粒子的密度分布均匀
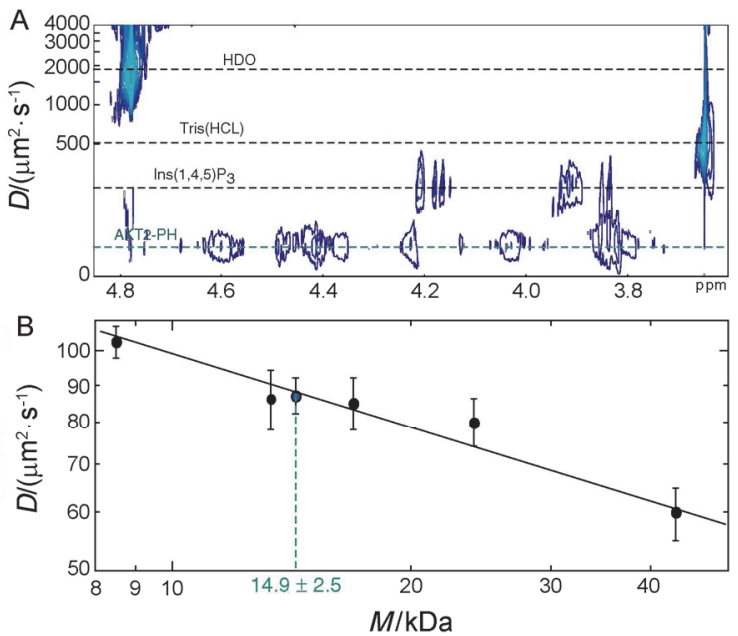

图 5 核磁共振法中扩散系数 $D$ 与重均分子量 $M_{\mathrm{w}}$ 之间的指数关系 Figure 5 Exponential relationships between the diffusion coefficient $(D)$ and the weight-average molecular weight $M_{\mathrm{w}}$ via the nuclear magnetic resonance (NMR) ${ }^{[25]}$ 
时，这一指数 $\delta$ 与 $v$ 是等同的. 这一指数关系已用于表 征溶液粒子的分形维数, 从而得到高分子单链或聚集体 在溶液中的形态结构 ${ }^{[28]}$.

\section{3 利用指数律研究高分子溶液中的单链构象}

高分子溶液中的单链构象反映了高分子单链在溶 液中的形态结构, 是高分子最重要的基本物理特征. 高 分子的链结构就像建筑物的基本材料一一砖瓦, 各具特 色的砖瓦决定了建筑物的基本性能; 同样地, 高分子的 链结构特征决定了高分子材料的基本性能 ${ }^{[3]}$. 因此, 研 究高分子单链的结构特征对于高分子材料的分子设计 及优异性能的高分子产品的开发具有重要指导意义 ${ }^{[29]}$. 这里, 我们将从高分子的不同种类切入, 介绍如何用指 数规律研究不同类型的高分子单链.

\section{1 天然生物类高分子}

目前, 许多人工合成的高分子材料因其高毒性, 难 降解给人们的生活带来了很大的危害. 天然类高分子由 于其取材方便、对环境友好、低毒、低危害、而且生物 活性好等优点得到了科学家们的高度重视, 相当多的天 然生物类高分子如多糖、蛋白质、DNA 等被研究并应 用于生物材料等领域. 而溶液中的链构象对此类高分子 生物活性和进一步加工应用影响重大. 因此, 关于此类 高分子在溶液中的链构象研究比较多, 利用指数规律研 究其高分子单链构象的报道亦多见 ${ }^{[30 \sim 37]}$.

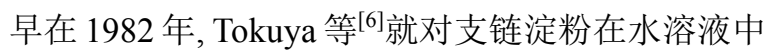
的链构象进行了研究. 他们利用沉淀分级的方法对原始 样品进行分级, 得到一系列窄分布的不同分子量样品, 然后利用黏度和光散射法分别得到了支链淀粉在水中 的 M-H(Mark-Houwink) 指数 $\alpha 0.5$; 和分形维数倒数 $\left(d_{\mathrm{f}}\right.$ $=1 / v) 0.57$, 依此确定其在溶液中的链构象是伸展的柔 性线团. Norisuye 等 ${ }^{[31]}$ 在 1984 年根据公式(2)研究了黄原 胶在 $0.1 \mathrm{~mol} / \mathrm{L}$ 的 $\mathrm{NaCl}$ 水溶液和氢氧化镉乙二胺溶液中 的链构象, 发现在 $M_{\mathrm{w}}$ 低于 $3 \times 10^{5}$ 时, 水溶液中的链构 象呈刚性; 而当高于此分子量时, 链构象呈半刚性. Cui 等 ${ }^{[32]}$ 利用黏度法和动静联用光散射的方法测定了小麦 $\beta$-葡聚糖单链在 $\mathrm{NaOH}$ 水溶液中的 $\mathrm{M}-\mathrm{H}$ 指数 $\alpha$ 、分形维 数倒数 $\left(d_{\mathrm{f}}\right)$ (如图 6 所示), 和形状参数 $\left(R_{\mathrm{g}} / R_{\mathrm{h}}\right)$, 这三个参 数相互验证. 最终得出结论: 小麦 $\beta$-葡聚糖在 $\mathrm{NaOH}$ 溶 液中呈现无规卷曲的链构象, 而且符合圆柱蠕虫模型.

张俐娜等 ${ }^{[33}$ 35] 利用指数规律研究了许多类型的多 糖类物质, 如 $\beta$-葡聚糖, 虎奶菇中提取的不溶于水的多 聚糖和竹节参多糖(如图 7 所示). 在这些研究中, 他们 都利用了黏度法和光散射法, 依据公式(1)和(2)研究了 这些多糖类物质的单链构象, 并得到这些物质在各自良 溶剂中的 $\mathrm{M}-\mathrm{H}$ 指数 $\alpha$ 和分形维数 $D$, 结合 TEM(透射电 镜)和 $\operatorname{AFM}$ (原子力显微镜), 最终判断它们在溶液中的
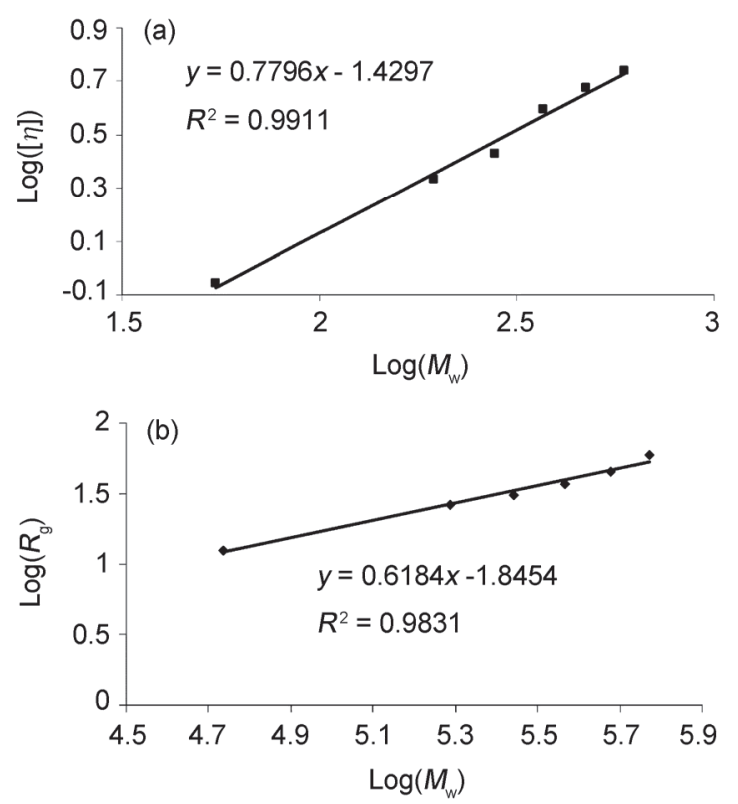

图 6 (a)小麦 $\beta$-葡聚糖在 $0.5 \mathrm{~mol} / \mathrm{L}$ 的 $\mathrm{NaOH}$ 溶液中特性黏度 $[\eta]$ 的对 数与重均分子量 $M_{\mathrm{w}}$ 之间的指数关系图. (b) 小麦 $\beta$-葡聚糖在 $0.5 \mathrm{~mol} / \mathrm{L}$ 的 $\mathrm{NaOH}$ 溶液中均方根回旋半径 $R_{\mathrm{g}}$ 的对数与重均分子量 $M_{\mathrm{w}}$ 之间的指 数关系图

Figure 6 (a) Double logarithmic plot of $[\eta]$ against $M_{\mathrm{w}}$ of wheat $\beta$-glucan in $0.5 \mathrm{~mol} / \mathrm{L} \mathrm{NaOH}$ solution. (b) Double logarithmic plot of the molecular weight $M_{\mathrm{w}}$ vs the radius of gyration $R_{\mathrm{g}}$ of wheat $\beta$-glucan in 0.5 $\mathrm{mol} / \mathrm{L} \mathrm{NaOH}$ solution $^{[32]}$
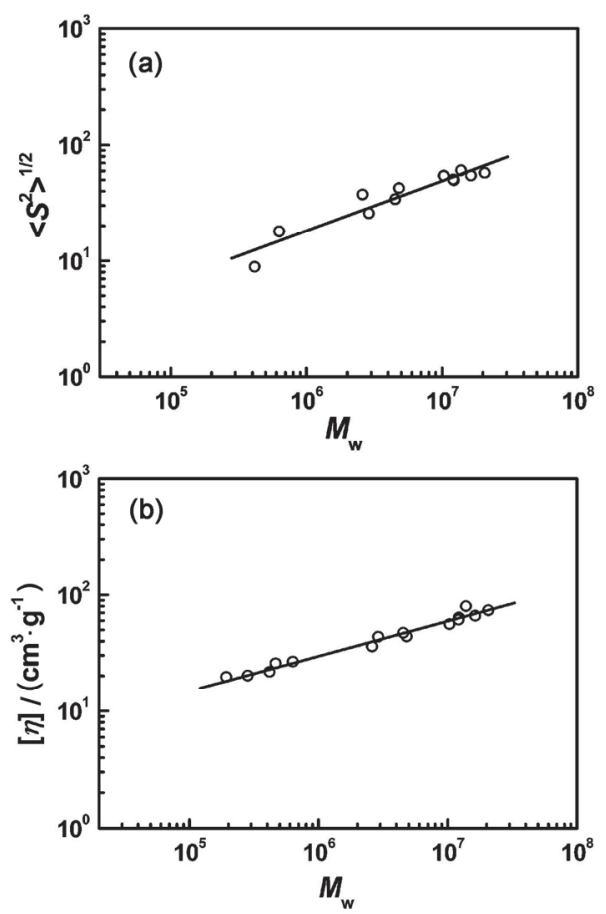

图 7 (a) 水溶性多聚糖(TM3a) 在 $0.25 \mathrm{~mol} / \mathrm{L}$ 的 $\mathrm{LiCl} / \mathrm{DMSO}$ 溶液中均 方根回旋半径 $\left\langle s^{2}>^{1 / 2}\left(R_{\mathrm{g}}\right)\right.$ 的对数与重均分子量 $M_{\mathrm{w}}$ 之间的指数关系图. (b) 水溶性多聚糖(TM3a) 在 $0.25 \mathrm{~mol} / \mathrm{L}$ 的 $\mathrm{LiCl} / \mathrm{DMSO}$ 中特性黏度 $[\eta]$ 的对数与重均分子量 $M_{\mathrm{w}}$ 之间的指数关系图

Figure 7 (a) Plot of $\log \left\langle\mathrm{s}^{2}\right\rangle^{1 / 2}\left(R_{\mathrm{g}}\right)$ vs $\log M_{\mathrm{w}}$ for TM3a in $0.25 \mathrm{~mol} / \mathrm{L}$ $\mathrm{LiCl} / \mathrm{DMSO}$. (b) Double logarithmic plot of $[\eta]$ against $M_{\mathrm{w}}$ of TM3a in $0.25 \mathrm{~mol} / \mathrm{L} \mathrm{LiCl} / \mathrm{DMSO}^{[33]}$ 
单链形状分别为刚性三螺旋构象、球状构象和球状链构 象. 研究中他们将 SEC(尺寸排除色谱)和 SLS(静态光 散射)联用, 从而更加快捷地得到了不同分子量多糖的 形状信息 ${ }^{[34,35]}$.

\section{2 超枝化高分子}

超枝化高分子是一类比较重要的高分子材料, 最早 产生于合成反应的副反应. 但近 30 年来, 超枝化高分子 越加受到人们的重视而成为合成高分子的重要部分. 原 因在于超枝化高分子可引入各类官能团并形成各式各 样的结构, 从而得到性质各异的高分子材料. 目前, 枝 化的控制已经成为设计、合成大分子功能材料的重要手 段 ${ }^{[36]}$. 然而, 如何表征合成产物的结构也成为了科学家 们关注的焦点. 超枝化高分子的结构虽然比较复杂, 但 其特性黏度、均方回旋半径等参数仍然与分子量有关. 因此, 利用指数规律可以表征超支化高分子单链的形 状，密度及自相似性.

对超支化高分子在溶液中的 M-H(Mark-Houwink) 指数 $\alpha$ 的研究已有很多报道. 结果显示: $\alpha$ 皆处于 $0.3 \sim$ 0.5 之间 ${ }^{[37]}$, 表明超支化大分子的形态结构介于二维盘 状和三维球状之间. 值得注意的是: 在这些研究中, 均 利用了 SEC 和黏度测定联用的装置 ${ }^{[37,38]}$ (如图 8 所示). 使得黏度和分子量之间的指数关系符合线性规律. 但是 对于树枝状大分子, 指数关系会逐渐偏离理想曲线. 原 因在于树枝状大分子分子量的增加将导致分子密度增 加以至于分子的均匀性下降 ${ }^{[39,40]}$, 使得 $\mathrm{M}-\mathrm{H}$ 指数随着 分子量增加而逐渐减小, 最终趋近于 0 , 即分子链会变 得越来越紧密. 有报道称: 当分子量达到 100000 时, 黏 度将会达到最大值.

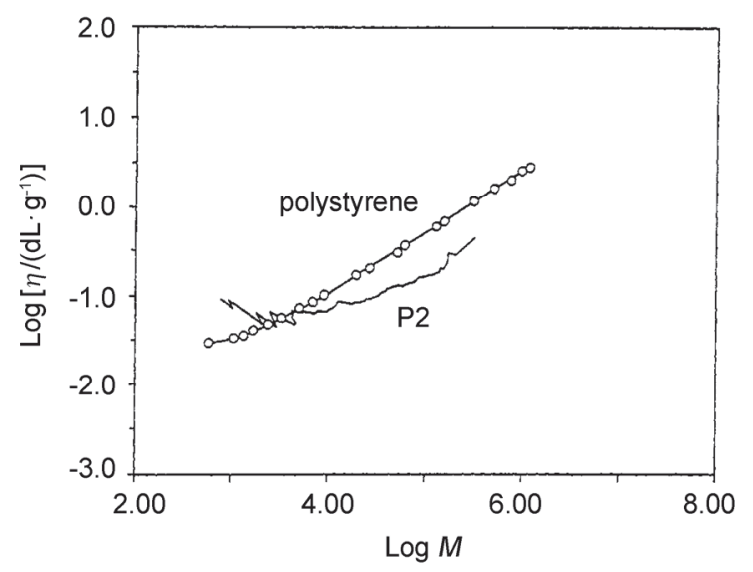

图 8 由 SEC 和黏度测定联用装置测定的两种超支化聚酯的特性黏度 $[\eta]$ 的对数与重均分子量 $M_{\mathrm{w}}$ 之间的指数关系图

Figure 8 Plot of $\log [\eta]$ vs $\log \left[M_{\mathrm{w}}\right]$ for two kinds of hyperbranched polyester as obtained from size-exclusion chromatography (SEC) \& viscosity determination device ${ }^{[38]}$

理论预测无规枝化高分子在良溶剂和 $\theta$ 溶剂中的 $d_{\mathrm{f}}$ 分别是 2.0 和 $2.28^{[41 \sim 43]}$, 而研究发现超支化高分子的 $d_{\mathrm{f}}$ 值一般在 $2 \sim 3$ 之间, 例如超支化聚酯的 $d_{\mathrm{f}}$ 是 $2.5^{[44]}$, 与
葡聚糖和淀粉的 $d_{\mathrm{f}}$ 值接近 ${ }^{[45,46]}$. 而理想的树枝状高分子 的 $d_{\mathrm{f}}$ 接近于 3 , 即接近于三维球体.

\section{3 共轭类聚合物}

共轭类聚合物是一类具有光电功能的材料, 在过去 的 30 年发展迅猛. 被广泛应用于聚合物发光二极管、有 机太阳能电池、激光发射器等领域. 目前报道大多集中 于材料合成、器件性能的提高方面, 但鲜见溶液中单链 形态的报道. 然而, 共轭聚合物在前体溶液中的形态与 其薄膜的聚集态结构及光电器件性能有本质上的联系, 已成为业内人士的共识. 因此, 研究共轭聚合物的单链 构象对调控其薄膜的凝聚态结构, 提高载流子迁移率及 其光电器件效率意义重大.

我们组 ${ }^{[47]}$ 研究了共轭聚合物聚 (9,9-二辛基芴) (PFO) 在氯仿中的单链 $\alpha$ 构象. 利用沉淀分级方法, 得到 窄分布的不同分子量 PFO 样品, 然后根据 $\mathrm{M}-\mathrm{H}$ 黏度方 程(1)得到了 $\mathrm{M}-\mathrm{H}$ 指数 $\alpha 1.44$; 结合形状参数 $R_{\mathrm{g}} / R_{\mathrm{h}}$, 判 断 PFO 在氯仿溶液中的链构象是半刚性疏松链. Knaapila 等 ${ }^{[48]}$ 则利用小角中子散射研究了侧链长度对 聚芴溶解性的影响. 根据公式(3)得到不同侧链长度聚 芴的光强-散射矢量之间的指数关系, 发现在不同的侧 链长度下, PF 衍生物的溶解性各不相同(如图 9): PF9 和 PF8 在甲苯溶液中的分形维数为 1 , 即表示可溶解成单 链; 但 PF7 和 PF6 的分形维数是 2, 则表示它们的溶解 性相对较差，会形成片状聚集.

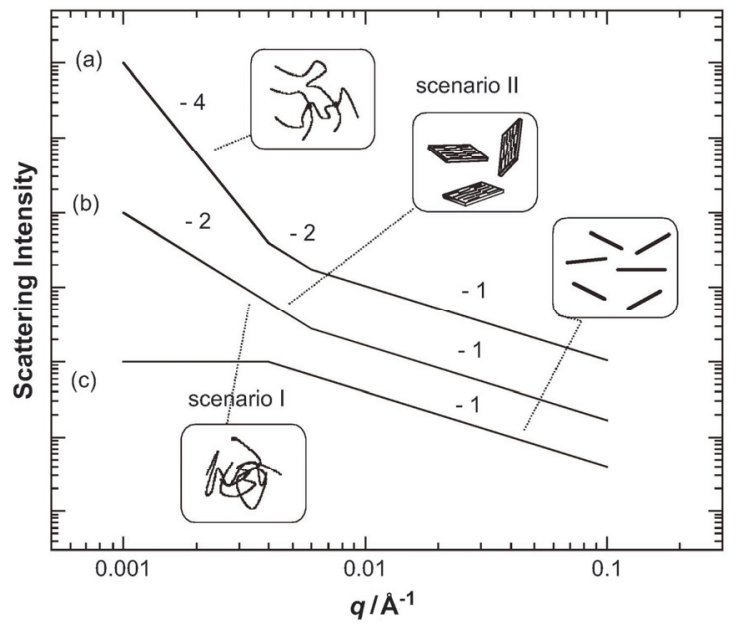

图9 由散射数据得到的光强 $I(q)$ 与散射矢量 $q$ 之间的指数关系, 得到 PF 衍生物在甲苯溶液中的结构: (a) 有交联点的网状结构; (b) 高斯线 团转变的棒状粒子(设想I)或者有节点的网状结构(设想 II); (c) 完全溶 解的棒状结构(实质是单链)

Figure 9 Schematic description of the scattering data and proposed structures of PFs in toluene: (a) a network-like structure with cross-linked nodes; (b) a structure of rodlike particles forming Gaussian coils (scenario I) or sheet-like aggregates (scenario II) in the longer length scales, and (c) a structure of fully dissolved rod like particles (essentially single polymer chains $)^{[48]}$

\section{4 用指数规律研究高分子溶液中的凝聚态结构}

高分子凝聚态结构是高分子溶液研究中的重要部 
分, 当高分子溶液的条件, 如温度、溶剂或者溶质浓度 发生改变时, 大量的单链会聚集, 从而形成不同形状和 尺寸的聚集体. 研究这些聚集体的凝聚态结构、尺寸、 特征及形成动力学, 对其在光电、生物、医药、材料等 领域应用意义重大. 然而, 一条条孤立的单分子链是如 何凝聚到一起的? 其单链与聚集态结构是否相同? 有 何不同? 其凝聚态的动力学演变过程的机制如何? 变 化规律如何? 迄今鲜见报道. 指数规律恰是一种用于研 究凝聚态结构的简单、有效的方法. 其中分形维数可用 以表征溶液中凝聚体的形状和尺寸. 因此, 非常适用于 研究高分子溶液的聚集行为.

\section{1 凝聚态结构的研究}

一般可以通过静态光散射法(SLS), 利用公式(3)得 到聚集体的分形维数 $d_{\mathrm{f}}$. 这一参数反映了聚集体的形态 和密实程度. 通过分子链运动模式的变化, 可研究聚集 态形成的动力学演变过程以及影响因素.

指数规律已用于不同类型高分子溶液的聚集体研 究, 如天然高分子凝胶、嵌段聚合物自组装聚集、共轭 聚合物凝胶等 ${ }^{[49 ~ 54]}$. de Spirito 等 ${ }^{[49]}$ 利用指数律研究了 纤维蛋白、纤维凝胶在缓冲溶液中形态结构随 $\mathrm{Cl}^{-}$的变 化过程. 发现随着 $\mathrm{Cl}^{-}$浓度增加, 分形维数从 1 增加到 1.8 , 证明聚集体随着离子浓度的增加变得越来越膨胀、 疏松 (随分形维数增大, 分子链凝聚的密实程度提高). 董宝中等 ${ }^{[24]}$ 利用小角 $\mathrm{X}$ 射线散射法研究了两亲性嵌段 聚合物 PSm- $b$-PVBAn 的聚集行为(如图 10 所示), 发现 质量分形维数 $D_{\mathrm{m}}$ 随着溶剂改变从 2.12 增加到 2.47 , 表 明聚集从溶胀线团变成相当密实的盘状结构. 而表面分 形维数 $D_{\mathrm{s}}$ 从 2.98 降至 2.58 , 证明聚集体的表面积由粗 粘变平滑; 而再加入 $\mathrm{CoCl}_{2}$ 之后分形维数明显增大, 证 明聚集体逐渐收缩成密实、且表面粗䊁的二维盘状结构. 陈信龙等 ${ }^{[50]}$ 和 Knaapila 等 ${ }^{[22]}$ 利用小角中子散射法

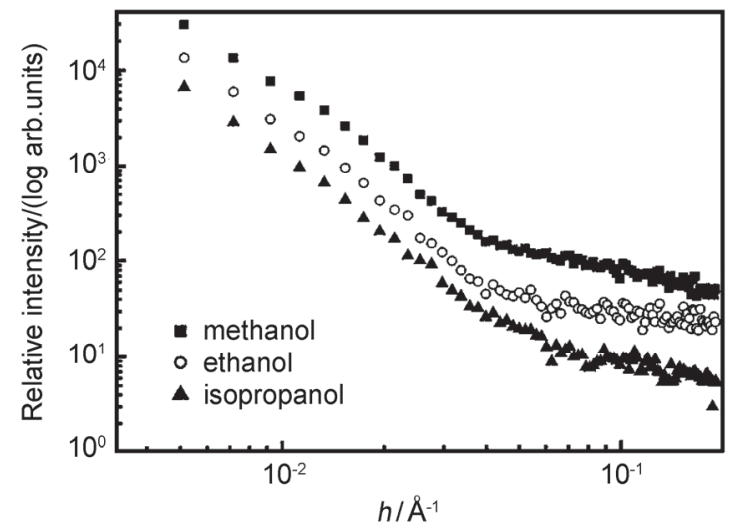

图 10 两亲性嵌段聚合物 $\mathrm{PS}_{68}-b-\mathrm{PVBA}_{38}$ 在不同溶剂条件下的 SAXS 图, 横坐标 $I(h)$ 是散射强度, 纵坐标 $h$ 是散射矢量. 后半段的曲线斜率 代表了聚集体的幂指数

Figure 10 SAXS curves of amphiphilic block copolymer $\mathrm{PS}_{68}-b$ $\mathrm{PVBA}_{38}$ in methanol, ethanol and isopropanol solvent. $I(h)$ is the scattering intensity and $h$ is the scattering vector. The right half of the curve slope represents the power index of aggregation ${ }^{[24]}$
(SANS)证明了共轭类聚合物 DP6-PPV 和三嵌段聚合物 PF/PANI11112- $b$-PANI11 在溶液中的聚集(其分形维数 $d_{\mathrm{f}}$ 在 $2.2 \sim 2.7$ 和 $2.2 \sim 2.8$ 之间)为网状结构. Bauer 等 ${ }^{[51]}$ 则 用 SANS 研究了碳纳米管聚集, 发现其聚集体的分形维 数 $d_{\mathrm{f}}=2.5$. Callejas-Fernández 等 ${ }^{[52]}$ 则利用光散射法表征 了聚乙烯基己内酰胺(PVCL)在水中形成的纳米颗粒凝 胶, 得到这种纳米颗粒是外壳粗粗、平均分形维数为 2.5、内核密度分布均匀的核-壳型球体结构. Muthukumar 等 ${ }^{[53]}$ 利用光散射研究了聚电解质聚磺苯乙烯的钠盐 $\mathrm{NaPSS}$ 在 $\mathrm{BaCl}_{2}$ 溶液中相分离的过程, 发现单链的分形 维数 $d_{\mathrm{f}}$ 是 $5 / 3$, 说明单链呈现伸展的链构象; 而聚集体 的分形维数 $d_{\mathrm{f}}$ 是 3.5 , 表明其分子链排列、堆砌的较密 实. 以上研究充分证明: 指数律是适用于各种高分子聚 集形态研究的理论工具.

\section{2 凝聚态结构动力学过程的研究}

凝聚态动力学过程的研究, 对认识其动力学演变过 程及规律、实现对凝聚态结构调控、从高分子本征性质 上提高材料与器件性能意义重大. 人们对于高分子的聚 集过程研究已有很多报道, 并提出了很多模型. 但到目 前为止，最被认可的理论模型有两种：DLCA(扩散限制 聚集模型 $)^{[55 \sim 57]}$ 与 $\operatorname{RLCA}$ (反应限制聚集模型) ${ }^{[58 \sim 61]}$. 在 DLCA 过程中, 扩散运动是聚集的唯一限制条件. 这一 模型假设粒子之间没有排斥作用, 粒子之间的每一次碰 撞都会导致不可逆的相互黏附. 因此, 这一碰撞过程往 往会形成较为疏松的聚集形态; 而在 RLCA 模型中, 由 于粘附几率比较小, 以至于粒子之间要碰撞很多次才能 相互黏附. 因此粒子有更多机会填充进聚集之中，这一 模型下产生的聚集往往会更加密实. 这两个过程形成的 聚集形状均可用分形维数这一特殊指数来描述. RLCA 过程的标志性分形维数 $\alpha$ 在 $2.1 \sim 2.2$ 之间 ${ }^{[61]}$, 而 DLCA 过程的分形维数为 $1.7 \sim 1.8$, 而且在聚集过程中聚集的 流体力学半径 $R_{\mathrm{h}}$ 与时间 $t$ 存在指数关系: $R_{\mathrm{h}} \propto t^{\alpha}$, 其中 $\alpha$ $<1^{[55]}$. 因此, 通过测定高分子溶液中聚集体的分形维 数, 可描述高分子溶液中聚集的形成过程, 从而推断凝 聚态结构的形成机理.

此方面研究已经有很多报道 ${ }^{[62 ~ 68]}$. 严大东等 ${ }^{[62]}$ 发 现间同立构甲基聚丙烯酸甲酯 $(s-\mathrm{PMMA})$ 在 $\theta$ 温度以下 的聚集分形维数为 2.1 , 符合 RLCA 过程. 而吴奇等 ${ }^{[63]}$ 的研究发现, 嵌段聚合物聚丙烯酸- $b$ - 聚甲基丙烯酸甲 酯(PAA- $b$-PNIPAM)在 $\mathrm{Ca}^{2+}$ 和加热情况下的聚集过程符 合 DLCA 模型. 更多研究发现, 溶液中的聚集方式是可 以调控的, 如 Kumagai 等 ${ }^{[64]}$ 的研究发现, 通过调节溶液 的温度和 $\mathrm{pH}$, 便可使牛血清蛋白(BSA)在溶液中的聚集 方式发生改变(如图 11 所示). 改变溶液环境, 如离子浓 度/种类、温度、 $\mathrm{pH}$ 等条件都可能使溶液中的聚集过程

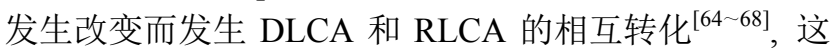
说明高分子的聚集过程是随环境变化的. 因此, 在特定 环境下的凝聚过程研究非常必要. 依据指数规律, 我们 
可以认识不同条件下高分子凝聚态的形成机理，从而调 控聚集态结构动力学演变过程, 获得我们期望的材料性 能.
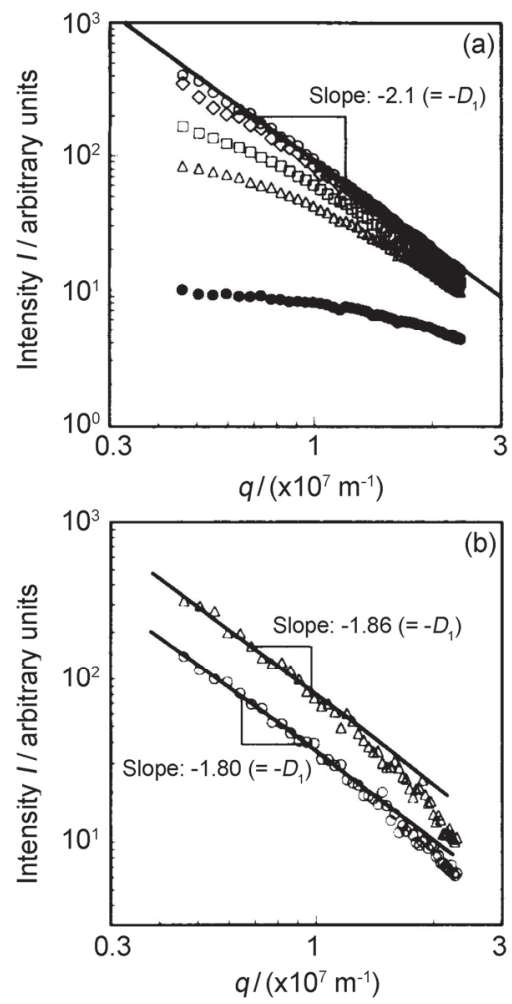

图 11 牛血清蛋白(BSA)溶液在不同 $\mathrm{pH}$ 和加热时间条件下的散射强 度 $I(q)$ 与散射矢量 $q$ 的双对数图. (a)聚集的分型维数 $d_{\mathrm{f}}$ 大概为 2.11 , 属 RLCA 过程; (b)聚集的 $d_{\mathrm{f}}$ 约为 1.8 , 属 DLCA 过程

Figure 11 Double logarithmic plot of scattered sight intensities $I$ of BSA solutions heated for various times $t_{a}$ against scattering vector $q$. (a) The fractal dimension of aggregation was about 2.11, which belongs to the RLCA process; (b) The fractal dimension of aggregation was about 1.8 , which belongs to the DLCA process ${ }^{[64]}$

\section{5 结论与展望}

高分子的物理性质是呈非线性的. 基于高分子链非 线性特征, 利用指数规律 (包括标度律) 去深刻认识高分 子的本征性质、运动规律, 单链、凝聚态相转变动力学 过程与机理意义重大. 随着越来越多功能高分子材料被 开发利用, 对其基本物理性质的认识尤为重要. 而指数 律不失为一种简便、有效的理论工具.

然而, 近十几年来, 基于高分子溶液中分子链本征 性质的基础研究仍不多见; 大部分工作集中于高分子材 料合成表征与提高器件性能, 鲜有从前体溶液的本征性 质切入的深入研究. 加之已有的标度律理论本身是基于 单链, 尚缺少在聚集态方面的理论支撑而主要由经验公 式代替. 对此, 亟待完善与创建. 加强高分子溶液的基 础研究将是未来的重要任务.
作者简介

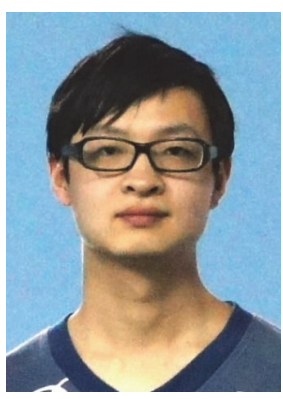

李涛, 吉林大学化学学院在读博士生. 2012 年毕业于华中 科技大学化学与化工学院并获得理学学士学位. 同年 9 月进 入吉林大学化学学院攻读博士研究生, 师从陆丹教授. 主要 应用静/动联用光散射技术, 从事共轭高分子在溶液中单链、 凝聚态结构形成及动力学演变过程的研究.

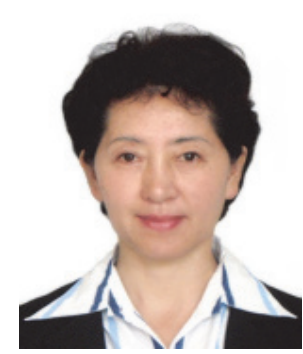

陆丹, 理学博士, 教授, 博士生导师. 1993 年考入中国科 学院长春应用化学研究所, 高分子化学与物理国家重点实验 室攻读硕士学位后直博. 师从于冯之榴、李滨耀研究员, 1998 年获得博士学位. 1998 2001 年, 在吉林大学超分子结构与材 料国家重点实验室, 师从于沈家骢院士做博士后研究工作, 后留校任教. 研究兴趣主要包括: 共轭高分子链构象变化对 载流子迁移特性影响的研究; 外电场对共轭高分子有序结构 的调控及载流子迁移特性的影响; 共轭高分子 PFO 链构象及 其凝聚态结构的标度律研究.

\section{References}

[1] Wu, Q. Y. Polymer Condensed Matter Physics, Science Press, Beijing, 2012, pp. 32 44. (吴其晔, 高分子凝聚态物理学, 科学出版 社, 北京, 2012, pp. 32 44.)

[2] de Gennes, P. E. Scaling Concepts in Polymer Physics, Cornell University Press, New York, 1985.

[3] Wu, Q. Y. China Plastics 2013, 27(1), 1. (吴其晔，中国塑料, 2013, 27(1), 1.)

[4] Robinson, G.; Ross-Murphy, S. B.; Morris, E R. Carbohydr. Res. 1982, 107, 17.

[5] Teraoka, I. Polymer Solutions: An Introduction to Physical Properties, John Wiley \& Sons, Inc., New York, 2001, pp. 209 221.

[6] Kato, T.; Okamoto, T.; Tokuya, T.; Takahashi, A. Biopolymers 1982, $21,1623$.

[7] Picton, L.; Bataille, G.; Muller, G. Carbohydr. Polym. 2000, 42, 23.

[8] Picton, L.; Merle, L.; Muller, G. Int. J. Polym. Anal. Ch. 1996, 2 , 103.

[9] Beaucage, G. Phys. Rev. E 2004, 70, 031401.

[10] Gelade, E. T. F.; Goderis, B.; de Koster, C. G..; Meijerink, N.; van Benthem, R. A. T. M. Macromolecules 2001, 34, 3552.

[11] Scherrenberg, R.; Coussens, B.; Van Vliet, P.; Edouard, G.; Brackman, J.; De Brabander, E. Macromolecules 1998, 31, 456.

[12] Huber, K.; Witte, T.; Hollmann, J.; Keuker-Baumann, S. J. Am. Chem. Soc. 2007, 129, 1089.

[13] Lages, S.; Michels, R.; Huber, K. Macromolecules 2010, 43, 3027.

[14] Carpinti, M.; Ferri, F.; Giglio, M.; Paganini, E.; Perini, U. Phys. Rev. A 1990, 42, 7347. 
[15] Schärtl, W. Light Scattering from Polymer Solutions and Nanoparticle Dispersions, Springer Laboratory, Berlin, 2007.

[16] Raspaud, E.; Lairez, D.; Adam, M.; Carton, J. P. Macromolecules 1994, 27, 2956.

[17] Peng, S. F.; Wu, C. Macromolecules, 2001, 34, 6795.

[18] Roe, R. J. Methods of X-ray and Neutron Scattering in Polymer Science, Oxford, New York, 2000.

[19] Higgins, J. S.; Benoit, H. C. Polymers and Neutron Scattering, Oxford, New York, 1994.

[20] Perahia, D.; Traiphol, R.; Bunz, U. H. F. J. Chem. Phys. 2002, 117, 1827.

[21] Wang, H.; Zhou, W.; Ho, D. L.; Winey, K. I.; Fischer, J. E.; Glinka, C. J.; Hobbie, E. K. Nano Lett. 2004, 4, 1789.

[22] Knaapila, M.; Garamus, V. M.; Alma'sy, L.; Pang, J. S.; Forster, M.; Gutacker, A.; Scherf, U.; Monkman, A. P. J. Phys. Chem. B 2008, $112,16415$.

[23] Perahia, D.; Jiao, X. S.; Traiphol. R. J. Polym. Sci. Polom. Phys. 2004, 42, 3165.

[24] Rong, L. X.; Wei, L. H.; Dong, B. Z.; Hong, X. G.; Li, F. M.; Li, Z. C. Chin. Phys. 2003, 12, 771. (荣利霞, 魏柳禾, 董宝中, 洪新国, 李福绵, 李子臣, 中国物理, 2003, 12, 771.)

[25] Auguin, D.; Gostan, T.; Delsuc, M.-A.; Roumestand, C. C. $R$. Chimie 2004, 7, 265.

[26] Crutchfield, C. A.; Harris, D. J. J. Magn. Reson. 2007, 185, 179.

[27] Auge, S.; Schmit, P.-O.; Crutchfield, C. A.; Islam, M. T.; Harris, D. J.; Durand, E.; Clemancey, M.; Quoineaud, A. A.; Lancelin, J. M.; Prigent, Y.; Taulelle, F.; Delsuc, M. A. J. Phys. Chem. B 2009, 113, 1914.

[28] Chari, K.; Antalek, B.; Minter, J. Phys. Rev. Lett. 1995, 74, 3624.

[29] Wu, Q. Y. Polymer Physics, Higher Education Press, Beijing, 2011, pp. $17 \sim 24$. (吴其晔, 高分子物理学, 高等教育出版社, 北京, 2011, pp. 17 24.)

[30] Roubroeks, J. P.; Mastromauro, D. I.; Andersson, R.; Christensen, B. E.; Åman, P. Biomacromolecules 2000, 1, 584.

[31] Sato, T.; Norisuye, T.; Fujita, H. Macromolecules 1984, 7, 6.

[32] Li, W.; Cui, S. W.; Wang, Q. Biomacromolecules 2006, 7, 446.

[33] Tao, Y. Z.; Zhang, L. N.; Yan, F.; Wu, X. J. Biomacromolecules 2007, 8, 2321.

[34] Huang, Z. P.; Huang, Y. N.; Li, X. B.; Zhang, L. N. Carbohydr. Polym. 2009, 78, 596.

[35] Li, S.; Huang, Y.; Wang, S.; Xu, X. J.; Zhang, L. N. J. Phys. Chem. $B$ 2014, 118, 668 .

[36] Voit, B. I.; Albena, L. Chem. Rev. 2009, 109, 5924.

[37] Mori, H.; Müller, A. H. E.; Simon, P. F. W. In Macromolecular Engineering: Precise Synthesis, Materials Properties, Applications, Vol. 2, Eds.: Matyjaszewski, K.; Gnanou, Y.; Leibler, L., Wiley-VCH, Weinheim, Germany, 2007, p. 973.

[38] Turner, S. R.; Voit, B. I.; Mourey, T. H. Macromolecules 1993, 26, 4617.

[39] Mourey, T. H.; Turner, S. R.; Rubinstein, M.; Fre'chet, J. M. J.;
Hawker, C. J.; Wooley, K. L. Macromolecules 1992, 25, 2401.

[40] Tomalia, D. A.; Hedstrand, D. M.; Wilson, L. R. In Encyclopedia of Polymers Science, 2nd ed., Wiley, New York, 1990.

[41] Isaacson, J.; Lubensky, T. C. J. Phys. Lett. 1980, 41, 469.

[42] Daoud, M.; Joanny, J. F. J. Phys. (Les Ulis, Fr.), 1981, 42, 1359.

[43] Flory, P. J. Principles of Polymer Chemistry, Cornell University, Press, Ithaca, New York, 1953.

[44] Luca, E. D.; Richards, R. W.; Grillo, I.; King, S. M. J. Polym. Sci. Polom. Phys. 2003, 41, 1352.

[45] Ioan, C. E.; Aberle, T.; Burchard, W. Macromolecules 2000, 33, 5730 .

[46] Hanselmann, R.; Burchard, W.; Lemmes, R.; Schwengers, D. Macromol. Chem. Phys. 1995, 196, 2259.

[47] Huang, L.; Zhang, L. L.; Huang, X. N.; Li, T.; Liu, B.; Lu, D. J. Phys. Chem. B. 2014, 118, 791.

[48] Knaapila, M.; Almásy, L.; Garamus, V. M.; Ramosd, M. L.; Justino, L. L. G.; Galbrecht, F.; Preis, E.; Scherf, U.; Burrowsd, H. D.; Monkmanm, A. P. Polymer 2008, 49, 2033.

[49] Papi, M.; Arcovito, G.; de, Spirito. M..; Amiconi, G.; Bellelli, A.; Boumis, G. Appl. Phys. Lett. 2005, 86, 183901.

[50] Li, Y. C, Chen, K. B, Chen, H. L.; Hsu, C. S.; Tsao, C. S.; Chen, J. H.; Chen, S. A. Langmuir 2006, 22, 11009.

[51] Bauer, B. J.; Hobbie, E. K.; Becker, M. L. Macromolecules 2006, 39, 2637.

[52] Callejas-Fernández, J.; Ramos, J.; Forcada, J.; Moncho-Jordá, A. J. Colloid Interface Sci. 2015, 450, 310.

[53] Kanai, S.; Muthukumar, M. J. Chem. Phys. 2007, 127, 25.

[54] Dai, S.; Tam, K. C.; Jenkins, R. D. Macromolecules 2000, 33, 404.

[55] Witten, T. A.; Sander, L. M. Phys. Rev. Lett. 1981, 47, 1400.

[56] Witten, T. A.; Sander, L. M. Phys. Rev. B 1983, 27, 5686.

[57] Meakin, P. Phys. Rev. Lett. 1983, 51, 1119.

[58] Meakin, P. Phys. Rev. A 1990, 41, 2005.

[59] Meakin, P. Adv. Colloid Interface Sci. 1988, 28, 249.

[60] Brown, W. D.; Ball, R. C. J. Phys. A 1985, 18, 517.

[61] Vicsek, T. Fractal Growth Phenomena, World Scientific, London, 1992.

[62] Chen, W. N.; Zhao, Y.; Jiang, Y.; Yan, D. D.; Han, C. C. ChemPhysChem 2004, 5, 1745.

[63] Liu, X. B.; Luo, S. K.; Ye, J.; Wu, C. Macromolecules 2012, 45, 4830.

[64] Hagiwara, T.; Kumagai, H.; Nakamura, K. Biosci. Biotech. Biochem. 1996, 60, 1757.

[65] Lin, W.; Zhou, Y. S.; Zhao, Y.; Zhu, Q. S.; Wu, C. Macromolecules 2002, 35, 7407 .

[66] Liao, W.; Zhang, Y. J.; Guan, Y.; Zhu, X. X. Langmuir 2012, 28, 10873.

[67] Burns, J. L.; Yan, Y. D.; Jameson, G. J.; Biggs, S. Langmuir 1997 $13,6413$.

[68] Li, N.; Li, Y. B.; Wang, X. G. Macromolecules 2011, 44, 8598.

(Cheng, B.) 